\title{
Adaptive Modulation and Coding based Error Resilience for Transmission of Compressed Video
}

\author{
Ryan Perera, Anil Fernando, Hemantha K. Arachchi \\ Centre for Vision Speech and Signal Processing \\ Department of Electronic Engineering \\ University of Surrey \\ United Kingdom \\ \{g.perera, w.fernando, h.kodikaraarachchi\}@surrey.ac.uk
}

\author{
Muhammad Ali Imran \\ Institute for Communication Systems \\ Department of Electronic Engineering \\ University of Surrey \\ United Kingdom \\ m.imran@surrey.ac.uk
}

\begin{abstract}
High spectral efficiency is at the core of any effort made to achieve the data rates and capacity requirements demanded by next generation mobile technologies. Adaptive Modulation and Coding (AMC) is considered a powerful tool for the efficient usage of spectrum and for the provision of channel adaptive error resilience. In this paper, a novel AMC based transmission scheme is introduced for Long Term EvolutionAdvanced (LTE-A) networks to impose adaptive error resilience on High Efficiency Video Coding (HEVC) based video data at the physical layer. A model is proposed to rank REs at the LTE-A transmitter based on channel information. Data are allocated onto ranked REs to maximise the efficiency of the users' allocated bandwidth while maintaining video quality. This is achieved by imposing a stronger Modulation and Coding Scheme (MCS) for data that have a high probability of being distorted, thus spanning the entire allocated bandwidth. Simulation results show that the proposed Unequal Error Protection (UEP) strategy helps to minimise the LTE-A transmitter power by up to $8 \mathrm{~dB}$ with guaranteed video quality. In effect, this is a reduction of approximately $84 \%$ of the transmit power.
\end{abstract}

Keywords- AMC; channel estimation; error resilience; HEVC; LTE-A; OFDM; QoS; unequal error protection

\section{INTRODUCTION}

Recently, everyday multimedia services such as teleconferencing, mobile television and peer-to-peer video sharing have undergone unprecedented growth. Many researches are now focused on catering to this demand as it poses a huge challenge, for wireless networks in particular, since the spectrum capacity is limited. Although the latest mobile communication standard LTE-A has helped to ease this situation by increasing downlink data rates to as high as $1.5 \mathrm{Gbps}$ [1], bandwidth still remains the main bottleneck. Therefore, when considering video delivery, effective means of compression are an important aspect. A common issue with many compression technologies such as HEVC [2] is that they give little emphasis on the transmission aspects of video data. A single transmission error can create artefacts in the reconstructed video frames and propagate in both spatial and temporal domains due to the hierarchical prediction scheme employed at compression. As a remedy error resilience measures are applied trading off on the radio resources utilized.

Among the many approaches addressing error resilience in compressed video transmission over mobile telephony, cross layer methods that adapt to the type of content and channel conditions have proven to give the optimum solutions. Reference [3] used feedback from lower layers of the communication protocol stack to the source coding stage to adjust the reference picture selection and encoder rate control. The work presented in [4] reaffirms the use of cross layer feedback by demonstrating adaptive transmission rates for Scalable Video Coding layers in a H.264/AVC system. Although adaptive methods are desired, these technologies treat all data with equal priority at each layer of the protocol stack and as a result, packets containing both high priority information such as parameter sets, as well as low-priority information are equally vulnerable to channel distortions.

On the contrary, exploiting UEP methods, [5] presents a criteria to switch between spatial multiplexing and transmit diversity in a MIMO channel based on a frame-by-frame motion intensity metric and the receiver SNR. However, the switching decision is made once every Group-of-Pictures (GOP) ignoring micro features of the video sequence and therefore making it only suitable for slow fading channels. A more granular adaptation method, which can work in slice/slice-segments, is more suitable for optimising the network resources in a fast fading channel.

Another popular form of imposing UEP is the use of AMC [6], a promising technique to overcome dynamic fading in wireless communication. In [7] Low-Density Parity-Check codes and rotated constellations are used to protect important bits in a video compressed using multiple description coding. However, the techniques used therein are not in conformance with the specifications of modern wireless standards. An AMC algorithm, which exploits the quality variation of radio resources to match the prioritized data (base layer and enhancement layers), is presented in [8] for the transmission of scalable images. The authors however have not specified the chosen code rates, which may have resulted in too much redundant information. Moreover, they have considered BPSK as a candidate for modulation, which is not in conformance with LTE specifications, and the error concealment method used at the decoder is not discussed. It is unclear whether these factors had any bearing on the actual gains.

The main objective of this paper is to impose error resilience with minimal burden on the amount of radio resources (hereafter referred to as Resource Elements (REs), segments in the OFDM transmission grid onto which complex 


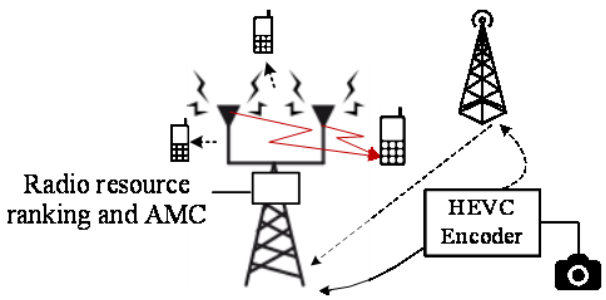

Fig. 1. Cellular downlink for video communication.

symbols can be assigned). We aim to use UEP on different parts of the video by exploiting the quality variation of REs. Initially, we modify the video stream such that data are sorted in their decreasing order of importance. Therefore, the aim translates to imposing more resilience onto the initial segments of the compressed data, input to the physical (PHY) layer.

Contrary to the conventional LTE systems, a main innovations of the proposed work is that it allows symbols of different MCSs to travel the wireless channel in harmony within one transmission time interval (TTI). Despite this feature, the proposal does not violate the standard AMC requirements of assigning a common MCS for each TTI at the PHY layer functions. The suggested approach makes use of the UE's existing reception and decoding functionalities with the addition of a simple preprocessing stage. Furthermore, the proposed algorithm uses all resources allocated to a particular user, thereby providing a more efficient technique.

As indicated in Fig. 1, this paper concerns a downlink where the type of data being transmitted is HEVC compressed video. Keeping focus on the objective, User Equipment (UE) management regarded outside the scope of this paper as it is an independent function. The algorithms presented have assumed that eNodeB allocates the entire bandwidth to one user at a given time interval of a radio frame. For performance evaluation peak signal to noise ratio (PSNR) is used because the transmitted data type is compressed video.

The rest of the paper is organized as follows. Section II illustrates the system model used while section III describes the proposed methodology. Simulation results are presented in section IV and section V contains the concluding remarks.

\section{REFERENCE SYSTEM MODEL}

The reference transmitter-receiver system inclusive of the proposed add-ons is presented in Fig. 2 and is illustrated in the following subsections. At eNodeB the HEVC coded sequence of video is prioritized to obtain the sequence $\hat{S}$. Based on the channel estimations of the previous radio frame the TBS Selection function determines the MCS to be used for each subframe in the subsequent radio frame.

Channel Coding and Modulation stage iterates for the 10 subframes and Symbol Buffer A accumulates all symbols for the following allocation radio frame. The Symbol Mapper matches the important symbols to the robust resources of the channel. The data are then transmitted through the channel one subframe at a time and the reverse operations are performed at the UE to recover the decoded video.

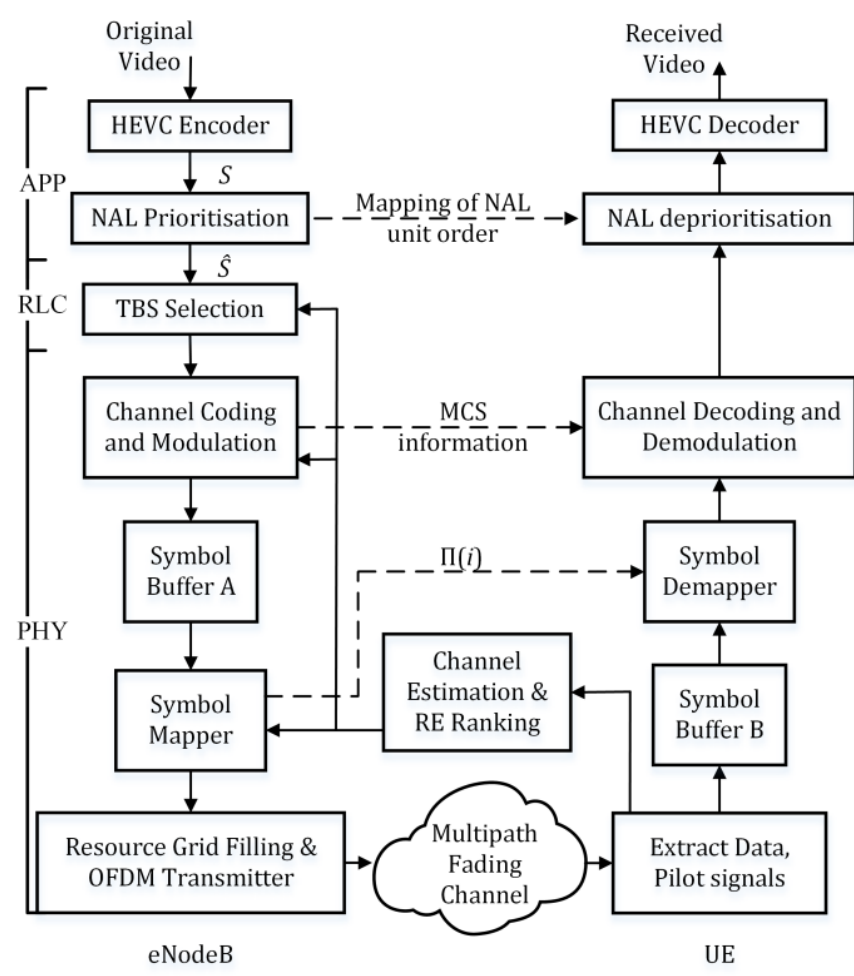

Fig. 2. End to end system.

\section{A. Video Sequence Prioritisation}

We consider a video stream of length $M$ number of frames compressed using a HEVC encoder. Each video frame is encoded into $Q$ number of slices. The video stream is a sequence of Network Abstraction Layer (NAL) units containing the parameter sets, intra coded NAL units and inter coded NAL units. A NAL unit is a string of compressed video that represents an independently decodable area of a frame known as a slice. Assume that the set of NAL units of a given $m^{\text {th }}$ video frame, $F_{m}$, is denoted as $\mathbf{N}_{m}$. If $N_{m, q} \in \mathbf{N}_{m}$ is the $q^{\text {th }}$ NAL unit of the input video frame, it can be denoted as a totally ordered set:

$$
F_{m}=\left\{N_{m, 1}, N_{m, 2}, \cdots, N_{m, q}, N_{m, q+1}, \cdots, N_{m, Q}\right\}
$$

where $T\left(N_{m, q}\right)<T\left(N_{m, q+1}\right)$ and $T($.$) denotes the arrival time.$ Subsequently, the input video sequence can be denoted by a similar totally ordered set,

$$
S=\left\{F_{1}, F_{2}, \cdots, F_{m}, F_{m+1}, \cdots, F_{M}\right\}
$$

where $T\left(F_{m}\right)<T\left(F_{m+1}\right)$.

In our proposed algorithm, the NAL units in each frame are sorted in the order of their importance in the prevention of error propagation within the decoded stream before the video sequence is output from the application (APP) layer. If $P(\cdot)$ denotes the importance, the sorted set of NAL units of $m^{\text {th }}$ frame can be denoted as the following totally ordered set:

$$
\hat{F}_{m}=\left\{\hat{N}_{m, 1}, \hat{N}_{m, 2}, \cdots, \hat{N}_{m, q}, \hat{N}_{m, q+1}, \cdots, \hat{N}_{m, Q}\right\}
$$


where $\hat{N}_{m, q} \in \mathbf{N}_{m}$ and $P\left(\hat{N}_{m, q}\right)>P\left(\hat{N}_{m, q+1}\right)$. As a result, the sorted video sequence becomes

$$
\hat{S}=\left\{\hat{F}_{1}, \hat{F}_{2}, \cdots, \hat{F}_{m}, \hat{F}_{m+1}, \cdots, \hat{F}_{M}\right\}
$$

where $T\left(\hat{F}_{m}\right)<T\left(\hat{F}_{m+1}\right)$.

The derivation of the importance of NAL units (i.e., $P($.$) )$ and the sorting procedure are presented in [9]. Mapping of NAL unit order from $F_{m}$ to $\hat{F}_{m}$ can be conveyed to the UE as a set of metadata via the Physical Downlink Control Channel (PDCCH).

This sorted sequence passes through the remaining OSI stack layers, accumulating header information, and is input to the RLC layer of eNodeB. In this study we consider the parameter sets to be transmitted error free. These are the initial three elements for sequence, picture and video parameter sets. We also allocate one video frame for each radio frame.

\section{B. TBS Selection}

An LTE-A standard compliant communication framework is used in this study. The choice of TB size is made at the RLC layer of eNodeB as indicated in Fig. 2. The PHY layer receives data as TBs, the size of which depends on the coding rate, $c_{j}$, the modulation choice, $m_{j}$, (where $m_{j}=2$ for QPSK, $m_{j}=4$ for 16QAM, etc.), and the number of PDSCH REs, $n_{j}$, of the $j^{\text {th }}$ subframe, where $j=\{1,2, . ., 10\}$. (Note that PDSCH is the set of user data carrying REs). As per the specifications of the LTE standard and the system configurations used within this study, $\Omega$ in (5) is chosen from the Table 7.1.7.2.1-1 of [10].

$$
T B S_{j}=\underset{x \in \Omega}{\arg \min }\left|\frac{x+24}{n_{j} m_{j}}-c_{j}\right|
$$

$$
n_{j}=(y \times a \times x-C S R-P S S-S S S-P B C H-P D C C H)
$$

where $a$ denotes the number of RBs in the allocated bandwidth (i.e., $1.4 \mathrm{MHz}$ ), CSR, PSS and SSS are the number of REs allocated for pilot symbols, primary and secondary synchronisation symbols respectively in the given subframe, $P B C H$ and $P D C C H$ are the number of broadcast REs and control channel REs. $y$ is the number of subcarriers within one $\mathrm{RB}$ (12 for LTE systems) and $x$ is the number of OFDM symbols of one subframe (typically 14). It should be noted that $P S S=0$ and $S S S=0$ for all subframes other than 1 and 6 , and $P B C H=0$ for all subframes except subframe 1 .

In the proposed technology data allocation is performed once every radio frame $(10 \mathrm{~ms})$. Hence, at the TBS Selection stage of Fig.2, eNodeB has complete knowledge of the quality of all radio resources of the subsequent radio frame. This knowledge is obtained from the Channel Estimation and RE Ranking function, where the REs are sorted in the descending order of their robustness. Based on the quality of the first portion of $n_{j \mid j=1}$ REs in the sorted RE set, an MCS is determined and $T B S_{j}$ selected accordingly. This is iterated for all 10 subframes.

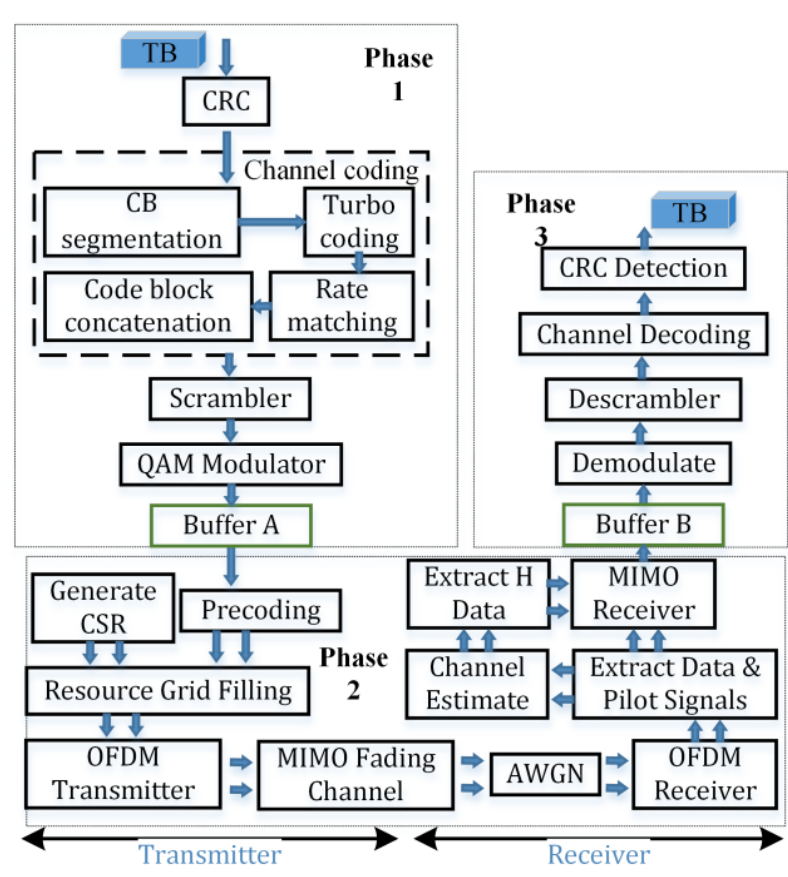

Fig. 3. PHY layer functions.

\section{PHY layer procedures}

The PHY layer functions are elaborated in Fig. 3. For clarity, these functions are presented in three phases. The purpose of Phase 1 is to fill Buffer A with complex data symbols for the subsequent allocation radio frame. Phase 2 transmits the data over the wireless channel, then received by the UE, and channel estimation is performed. Buffer B of Phase 3 accumulates the transmitted symbols of each subframe and the remaining UE functions are included in Phase 3.

Phase 1: After learning $T B S_{j}, m_{j}$ and $c_{j}$ to be used for each set of $n_{j}$ REs, TBs are taken from the bit stream and passed through Phase 1 in ten iterations. A Cyclic Redundancy Check is appended onto the TB and turbo coding is performed. The turbo coder consists of two recursive systematic convolutional encoders and outputs one systematic and two parity bit streams. These streams are interleaved, interlaced and placed on a circular buffer. Based on $c_{j}$, puncturing is automatically performed when reading from the circular buffer. These data are then modulated using $m_{j}$ (the system allows three modulation techniques; QPSK 16QAM and 64QAM).

Buffer A accumulates the complex symbols, one radio frame at a time. The resulting first set of $n_{j \mid j=1}$ symbols is of a higher order MCS, gradually decreasing towards lower orders for the $10^{\text {th }}$ subframe. This is because the first set of symbols is to be allocated to the most robust REs.

Phase 2: Upon collecting the symbols of all 10 subframes, they are mapped to different positions within the OFDM grid such that the oldest symbols in Buffer A (i.e., those of higher order MCS) are allocated to the most robust REs. The transmission is thereafter performed one subframe at a time. 
At the receiver's end, channel estimation is performed by first reading the CSR symbols. These symbols are transmitted at specific locations in the OFDM resource grid as predefined symbols. The receiver calculates the ratio between the original and received CSR symbols to obtain the channel coefficients, which are then averaged along subcarriers within each subframe. These data are transmitted back to eNodeB where the PDSCH channel coefficients are obtained using linear interpolation. Assuming the channel is slowly varying we consider these interpolated values to be valid information for channel estimation at the subsequent allocation period.

Phase 3: The Buffer B accumulates the equalized symbols and performs demapping to account for the mapping process at Buffer A. Thereafter the same $10 \mathrm{MCS}$ values are used to obtain the TBs of 10 subframes.

The proposed algorithm in Fig. 3 runs once every radio frame. As an overview, the system has been segmented into three sections. Phase 1 and 3 run 10 iterations, once for each subframe. Resource grid filling is performed on a per radio frame basis. The remainder of Phase 2 until Buffer B operates without any alteration to the LTE-A standard specifications and has one transmission every one millisecond.

\section{Proposed UneQual ERror Protection APPROACH}

The UE performs channel estimation with the assistance of pilot symbols [10]. Based on the feedback received from UE, eNodeB calculates the success probability of each RE of the radio frame. It should be noted that in this implementation it is assumed the rankings computed for the previous radio frame (allocation interval) is also valid for the current allocation interval. Let the success probability values be $r_{1}, r_{2}, \ldots, r_{\tau}$, where $\tau=\sum_{j=1}^{10} n_{j}$. Here $n_{j}$ is the number of REs in the $j^{\text {th }}$ subframe. The success probability values are sorted in the decreasing order of robustness to form $\hat{r}_{1}, \hat{r}_{2}, \ldots, \hat{r}_{\tau}$. The mapping function $\Pi(i)$ is conveyed to the receiver and it follows $\hat{r}_{i}=r_{\Pi(i)}, i=1,2, \ldots, \tau$.

\section{A. RE Boundaries}

For each SNR level, a boundary RE, $B_{M C S_{k}}(S N R)$ in the ranked $\mathrm{RE}$ order is specified to indicate the point above which the REs show an acceptable BER for a given $M C S_{k}$. These boundaries are pre-defined lookup values based on simulated statistical data obtained by transmitting a known set of symbols, which spans the entire ranked allocation area (radio frame), and analysing the point the received symbols become erroneous. Table I presents extracts from the results of trials performed for each SNR value to obtain these thresholds. In this extract, the considered modulation is 16QAM and the code rate is 0.5 , for a $1.4 \mathrm{MHz}$ downlink. For $5 \mathrm{~dB}$ the errors were spread throughout the bit sequence, hence the boundary is 0 . For SNR values above $11 \mathrm{~dB}$ (inclusive) the number of errors was negligible, hence the boundary is the maximum number of REs. The relationship between the bit position and the number of REs occupied by the preceding bits is depicted in Table II.
TABLEI. EXTRACTS FROM SIMULATION RESULTS FOR BOUNDARY DETERMINATION

\begin{tabular}{|c|c|c|c|c|c|c|}
\hline SNR & \multicolumn{2}{|c|}{ 6dB } & \multicolumn{2}{|c|}{$7 \mathrm{~dB}$} & \multicolumn{2}{|c|}{ 8dB } \\
\hline Trial & $\alpha$ & $e$ & $\alpha$ & $e$ & $\alpha$ & $e$ \\
\hline 1 & 9948 & 776 & 11394 & 531 & 12946 & 306 \\
\hline 2 & 9853 & 777 & 11401 & 523 & 12947 & 288 \\
\hline 3 & 9850 & 750 & 12938 & 329 & 12938 & 303 \\
\hline 4 & 9870 & 760 & 11397 & 508 & 12941 & 267 \\
\hline \multirow[t]{2}{*}{5} & 9852 & 726 & 11406 & 520 & 12946 & 312 \\
\hline & \multicolumn{2}{|c|}{ 9dB } & \multicolumn{2}{|c|}{$10 \mathrm{~dB}$} & \multicolumn{2}{|c|}{ 11dB } \\
\hline 1 & 12939 & 282 & 12945 & 179 & 13133 & 16 \\
\hline 2 & 12938 & 275 & 12953 & 208 & & \\
\hline 3 & 12940 & 275 & 12950 & 210 & & \\
\hline 4 & 12952 & 243 & 12949 & 184 & 13108 & 59 \\
\hline 5 & 12938 & 237 & 12957 & 228 & & \\
\hline
\end{tabular}

$\alpha$ : The bit position where the first bit error occurs $e$ : Number of bit errors (out of 14480)

TABLE II. SUBFRAME CAPACITIES

\begin{tabular}{|c|c|c|c|c|}
\hline $\begin{array}{c}\text { Subframe } \\
(j)\end{array}$ & $\begin{array}{c}\text { Number of } \\
\text { REs }\left(n_{j}\right)\end{array}$ & $\begin{array}{c}\text { Cumulative } \\
\text { REs }\end{array}$ & $T B S_{j}$ & $\begin{array}{c}\text { Cumulative } \\
\text { data bits }\end{array}$ \\
\hline 1 & 384 & 384 & 936 & 936 \\
\hline 2 & 792 & 1176 & 1544 & 2480 \\
\hline 3 & 792 & 1968 & 1544 & 4024 \\
\hline 4 & 792 & 2760 & 1544 & 5568 \\
\hline 5 & 792 & 3552 & 1544 & 7112 \\
\hline 6 & 648 & 4200 & 1192 & 8304 \\
\hline 7 & 792 & 4992 & 1544 & 9848 \\
\hline 8 & 792 & 5784 & 1544 & 11392 \\
\hline 9 & 792 & 6576 & 1544 & 12936 \\
\hline 10 & 792 & 7368 & 1544 & 14480 \\
\hline
\end{tabular}

Although these boundaries change marginally with the number and severity of the channel's fading paths, simulation results show that these boundaries tend to be similar for a given type of multi-path propagation environment (e.g., urban, semi urban etc.). Therefore, for a given SNR, these boundaries can be pre-calculated. However, the REs will change their positions in the ranked list. Therefore, in practical implementations RE ranking must be performed once every allocation interval.

\section{B. Adaptive modulation and coding}

After ranking REs of a radio frame based on their survival probability, the conversion of data bits to complex symbols (Phase 1) can commence. The CQI-recommended MCS is chosen as the default $M C S_{0}$ to create the symbols falling onto the most robust REs. As an example if $M C S_{0}$ is $16 \mathrm{QAM}$ with a code rate of $0.5(16,0.5)$, other MCSs are defined as,

$$
M C S_{0}, M C S_{1}, M C S_{2}, \ldots=(16,0.5),(16,0.4),(16,0.3),(4,0.7),(4,0.6) \ldots
$$

For each $M C S_{k}$ there is a different set of ranked RE boundaries above which the data allocation gives an acceptable BER. An example of results obtained for $(16,0.5)$ for a $1.4 \mathrm{MHz}$ system is presented in Table III. A further safety margin of $10 \%$ is used to guarantee the end user QoS in the event of unexpected burst errors. The tabulated values are for a BER of $10 \%$.

$M C S_{0}$ is used for the first $l$ subframes where 
TABLE III.

RE BOUNDARIES FOR DIFFERENT SNRS

\begin{tabular}{|c|c|c|}
\hline SNR & Boundary & Boundary allowing $\mathbf{1 0} \%$ margin $B_{m_{0}}(S N R)$ \\
\hline$<6 \mathrm{~dB}$ & 0 & 0 \\
\hline $6 \mathrm{~dB}$ & 4992 & 4255 \\
\hline $7 \mathrm{~dB}$ & 5784 & 5047 \\
\hline $8 \mathrm{~dB}$ & 6576 & 5839 \\
\hline $9 \mathrm{~dB}$ & 6579 & 5842 \\
\hline $10 \mathrm{~dB}$ & 6581 & 5844 \\
\hline$>10 \mathrm{~dB}$ & $7368($ all $)$ & 6631 \\
\hline
\end{tabular}

$$
l=\underset{l^{\prime} \in[1,10]}{\arg \min }\left(B_{M C S_{0}}(S N R)-\sum_{j=1}^{l^{\prime}} n_{j}\right)
$$

while the argument being positive. The subsequent subframes use $M C S_{k}$ which satisfies

$$
k=\underset{k \in[1,2, . .]}{\arg \min }\left(B_{M C S_{k}}(S N R)-\sum_{j=1}^{p} n_{j}\right)
$$

while the argume $\mathrm{nt}$ is maintained positive. $p$ refers to the subframes $l+1$ to 10 .

Based on the chosen $M C S_{k}$ for each subframe the RLC layer determines the $T B S_{j}$ and passes to the PHY layer $T B S_{j}$ unconverted bits from $\hat{S}$ bit stream in each iteration. The sets of complex symbols from each iteration are accumulated at Buffer A. The symbols are automatically sorted in the order of their importance since they were input to Phase 1 as important data first. These buffered symbols, which contain $\left(n_{1}+\ldots+n_{10}\right)$ elements are then mapped onto the sorted REs. However, since it is specified in the LTE standard that data allocation to REs must happen sequentially and the physical position of REs cannot be altered, we change the positions of the symbols in Buffer A before they are sequentially allocated to the REs. To assist in this mapping process we use the mapping $\Pi(i)$. After this mapping process Phase 2 can be commenced.

A segment of $n_{j}$ symbols $(j=1,2, . .10)$ are taken out from Buffer A to be transmitted as the $j^{\text {th }}$ subframe. This segment of $n_{j}$ symbols can be of different MCSs due to the mapping process. Therefore this allows complex symbols of different MCSs to travel the wireless channel within one TTI. Buffer B accumulates the $n_{j}$ symbols of 10 transmission intervals (the radio frame) and performs the demapping using $\Pi(i)$ information. The Phase 3 then performs the decoding using similar parameters as at the channel encoder and obtains the sequence of NAL units. These are then reordered to their original sequence and forwarded to the HEVC decoder.

\section{Simulation RESUlts}

To demonstrate the effectiveness of the proposed technique three different video sequences, representing different motion characteristics (low, medium, high), are considered. Therefore, different Quantisation Parameters (QPs) are used to encode the video sequences to maintain the bit rate at a predetermined level. Accordingly, QP values of 25, 34 and 32 are chosen for news, stefan and mobile sequences, respectively. The test video sequences [11] are of
4:2:0 YUV format and are commonly used in video compression related researches.

The PHY layer illustrated in Fig. 3 was implemented using the MATLAB LTE-System Toolbox, which has been tested and validated [12]. The benchmark used is an adaptation of the UEP approach presented in [8] in a manner that is compliant with the HEVC standard and LTE-A transceivers. As LTE does not allow different modulation schemes between subcarriers during one TTI, we have adapted the benchmark to select one for each subframe. Accordingly, the channel flattening stage in [8] occurs not over the carriers, but over the subframes. In the benchmark system the QPs are selected such that the whole allocated bandwidth is utilised. Accordingly, QPs of 24, 33 and 32 are selected for news, stefan and mobile sequences respectively. During simulations, slice copying is used as the error concealment strategy at the HEVC decoder. Finally, PSNR of the distorted video sequence is calculated with reference to the original video sequence under different channel SNR for the proposed technique and the abovementioned benchmark system.

Fig. 4 illustrates the average PSNR for the three video sequences under different channel SNR values. Irrespective of

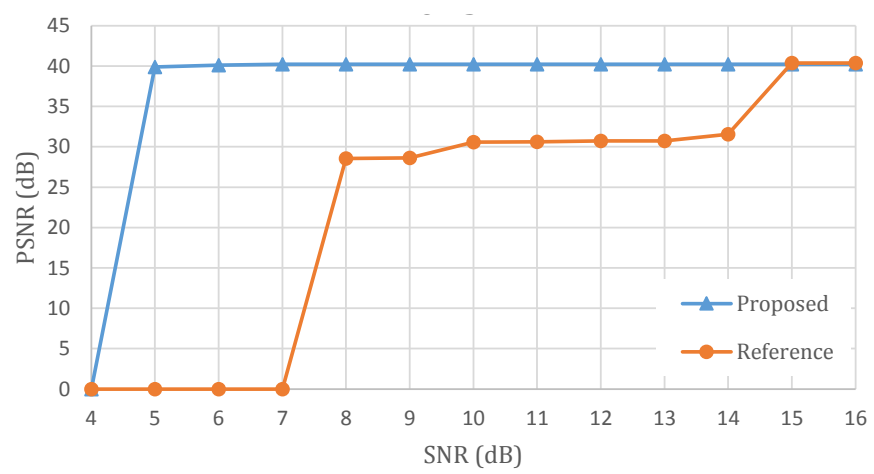

(a) News Sequence

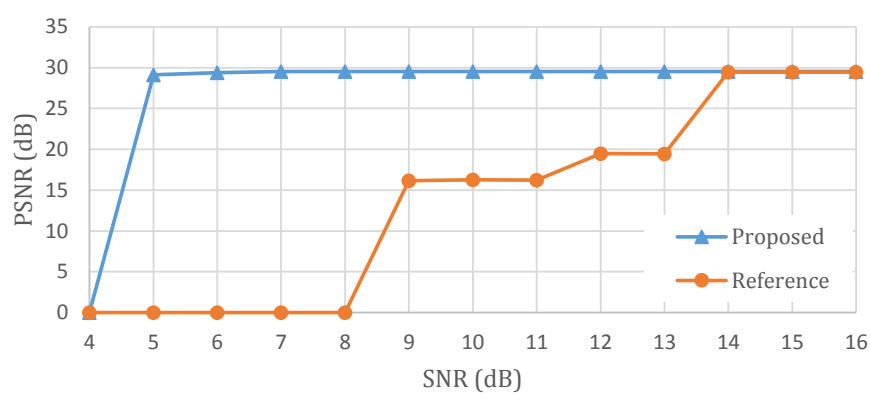

(b) Stefan Sequence

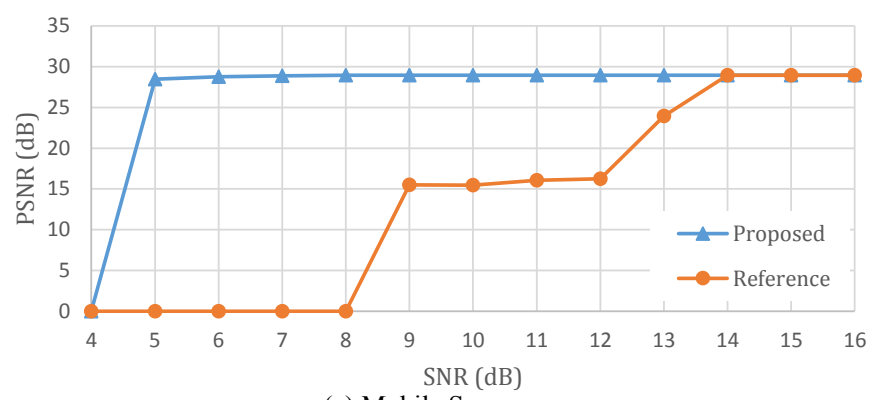

(c) Mobile Sequence

Fig. 4. Results 
the motion characteristics, all results show similar performance. The proposed system outperforms the conventional method of video communication up until $14 \mathrm{~dB}$ SNR. This is due to the sustainability of REs at lower SNR with the proposed system. In the benchmark, system performance is well below in comparison to the proposed system for all SNRs. The proposed system demonstrates an abrupt fall in PSNR as SNR is reduced. The AMC feature and the RE ranking algorithm collaboratively hold the PSNR at a superior level until all unused REs are exploited at $5 \mathrm{~dB}$.

Another obervation is that PSNR does not drop immediately below $13-14 \mathrm{~dB}$ in the benchmark system, but remains at a moderate value before failing entirely at $7-8 \mathrm{~dB}$. This is due to the deployed error concealment strategy, which recovers some of the distorted regions due to dropped slices. At SNR less than $5 \mathrm{~dB}$, both techniques have failed to decode any useful REs. This could have been improved with the proposed system if the quality of the video is sacrificed by increasing the QP size. By increasing the QP size the bit rate of the encoded video will decrease and therefore, in highly adverse channel conditions these data can be allocated to the reduced amount of REs caused by the adverse movement of the SNR threshold. However, this approach would require feedback between the PHY and APP layers, and will have practical limitations in real-time video communications.

Furthermore, results indicate that eNodeB signal power can be reduced up to $6 \mathrm{~dB}$ without noticeable degradation in the video quality. Therefore, the proposed technology can be considered as a significant step towards achieving real-time HEVC based video communications over LTE-A with minimum resources. Moreover, the proposed technology can be viewed as an enabling technology, which significantly increases the coverage radius of a given eNodeB with guaranteed PSNR.

The additional functions that are needed for the implementation of this algorithm over the commercially deployed LTE-A technology for video transmission are:

- Reordering the slices in the APP layer.

- $\quad$ PDCCH transmission of $F_{m}$ to $\hat{F}_{m}$ mapping.

- Buffering channel estimation values of 10 subframes.

- Sorting the robustness of REs.

- $\quad$ PDCCH transmission of these RE mappings ( $\Pi(i)$ ).

- Choosing the 10 MCS values from a lookup table.

- Symbol Buffer A (at eNodeB) and mapper.

- Symbol Buffer B (at UE) and demapper.

The sizes of Buffer A and B are $\left(n_{1}+. .+n_{j}+. .+n_{10}\right)$, and $n_{j}$ is as per (6). In our simulation setup this takes the value 7368. The sorting of REs is also performed on 7368 channel estimation values. This mapping information occupies trivial PDCCH overhead. The priority information of NAL units is also negligible, as the number of slices per video frame cannot be extremely large in order to maintain compression efficiency. This marginal increase in computational resources and PDCCH overhead are a negligible price to pay compared to the gains the proposed algorithms achieve.

\section{CONCLUSIONS}

In this paper, an efficient resource allocation scheme based on $\mathrm{RE}$ ranking and $\mathrm{AMC}$ is proposed for $\mathrm{HEVC}$ based real time video communications over LTE-A mobile networks. Based on channel estimation, REs are ranked according to their vulnerabilities to multi-path fading and noise for different SNRs. For each SNR an RE boundary is defined to indicate the transition $\mathrm{RE}$, until which the transmissions experience a predefined BER. Vulnerable REs below this boundary are protected with stronger MCS, taking advantage of any excess bandwidth allocated to the user. Simulation results show that the signal power can be reduced by $8 \mathrm{~dB}$ without noticeable video quality degradation. This result translates to a reduction of $84 \%$ of the signal power, trading off a marginal increase in the control channel data rate. Moreover, the additional computational overhead required for the proposed system is negligible compared to the gains obtained in the video transmission. Future work will focus on utilising spatial and temporal down sampling of HEVC video adaptively to further improve the system performance.

\section{REFERENCES}

[1] E. Dahlman, S. Parkvall, and J. Skold, $4 G$ LTE/LTE-Advanced for Mobile Broadband. Elsevier Ltd., 2011, pp. 143-149.

[2] G. J. Sullivan, J.-R. Ohm, W.-J. Han, and T. Wiegand, "Overview of the High Efficiency Video Coding (HEVC) Standard," IEEE Trans. Circuits Syst. Video Technol., vol. 22, no. 12, pp. 1649-1668, Dec. 2012.

[3] C. Zhang, Y. Wang, S. Zheng, and H. Zhang, "Multilevel Cross-Layer Feedback Based Adaptive Video Coding for Wireless Transmission," in 2012 8th International Conference on Wireless Communications, Networking and Mobile Computing, 2012, pp. 1-4.

[4] I. Ahmed, L. Badia, D. Munaretto, and M. Zorzi, "Analysis of PHY/application cross-layer optimization for scalable video transmission in cellular networks," in 2013 IEEE 14th International Symposium on WoWMoM), 2013, pp. 1-3.

[5] M. Hafeez, S. Jangsher, and S. A. Khayam, "A Cross-Layer Architecture for Motion-Adaptive Video Transmission over MIMO Channels," in 2011 IEEE International Conference on Communications (ICC), 2011, pp. 1-5

[6] J. Hayes, "Adaptive Feedback Communications," IEEE Trans. Commun., vol. 16, no. 1, pp. 29-34, Feb. 1968.

[7] C. C. Ru, L. G. Yin, J. H. Lu, and C. W. Chen, "A new UEP scheme based on adaptive modulation for robust video transmission in MIMO system," J. China Commun., vol. 3, no. 5, pp. 102-107, 2006.

[8] H. Houas, I. Fijalkow, and C. Baras, "Resources Allocation for the Transmission of Scalable Images on OFDM Systems," in 2009 IEEE International Conference on Communications, 2009, pp. 1-5.

[9] R. Perera, A. Fernando, T. Mallikarachchi, H. K. Arachchi, and M. Pourazad, "QoE aware resource allocation for video communications over LTE based mobile networks," 10th Int. Conf. Heterog. Netw. Qual. Reliab. Secur. Robustness, pp. 63-69, Aug. 2014.

[10] 3rd Generation Partnership Project (3GPP), "LTE; Evolved Universal Terrestrial Radio Access (E-UTRA); Physical layer procedures (3GPP TS 36.213 version 10.1.0 Release 10)," 2011.

[11] "YUV Sequences." [Online]. Available: http://trace.eas.asu.edu/yuv/index.html. [Accessed: 15-Apr-2015].

[12] "LTE System Toolbox Datasheet" [Online]. Available: http://uk.mathworks.com/products/datasheets/pdf/lte-systemtoolbox.pdf. [Accessed: 20-Apr-2015]. 